\title{
Geometry optimization of steroid sulfatase inhibitors - the influence on the free binding energy with STS
}

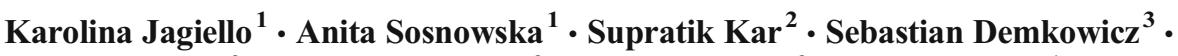 \\ Mateusz Daśko $^{3}$ • Jerzy Leszczynski ${ }^{2}$ - Janusz Rachon ${ }^{3} \cdot$ Tomasz Puzyn $^{1}$
}

Received: 2 November 2016 / Accepted: 19 December 2016/Published online: 7 January 2017

(C) The Author(s) 2017. This article is published with open access at Springerlink.com

\begin{abstract}
In the paper we review the application of two techniques (molecular mechanics and quantum mechanics) to study the influence of geometry optimization of the steroid sulfatase inhibitors on the values of descriptors coded their chemical structure and their free binding energy with the STS protein. We selected 22 STS-inhibitors and compared their structures optimized with MM+, PM7 and DFT B3LYP/6-31++G* approaches considering separately the bond lengths, angles, dihedral angles and total energies. We proved that different minimum energy conformers could be generated depending on the choice of the optimization method. However, the results indicated that selection of the geometry optimization method did not affect the optimal STS inhibitor coordinates, and hence the values of molecular descriptors which describe the 3D structure of the molecule. To study the interaction pattern of the STS inhibitors (optimized using different methods) with the target receptor we applied two strategies: AutoDock and PathDock. The docking studies point out that selection of software to docking simulation is one of the crucial factors determining the binding mode
\end{abstract}

Electronic supplementary material The online version of this article (doi:10.1007/s11224-016-0903-x) contains supplementary material, which is available to authorized users.

Tomasz Puzyn

t.puzyn@qsar.eu.org

1 Laboratory of Environmental Chemometrics, Faculty of Chemistry, University of Gdansk, Wita Stwosza 63, 80-308 Gdansk, Poland

2 Interdisciplinary Nanotoxicity Center, Department of Chemistry and Biochemistry, Jackson State University, 1400 JR Lynch Street, Jackson, MS 39217-0510, USA

3 Department of Organic Chemistry, Chemical Faculty, Gdansk University of Technology, Narutowicza 11/12, 80-233 Gdansk, Poland of STS inhibitors with their molecular target. Other factor is related to the ligand orientation in the binding pocket. Finally, obtained results indicate that MM+ and PM7 methods (faster and less expensive) could be successfully employed to geometry optimization of the STS inhibitors before their docking procedure as well as for molecular descriptors calculations.

Keywords Steroid sulfatase inhibitors - Geometry optimization - Molecular docking - Molecular mechanics . Quantum mechanics

\section{Introduction}

Over the past decades, numerous reports have suggested that the biologically active hormone precursors may affect on cellular proliferation in various cancers [1]. These compounds (including androgens and estrogens) play an important role in the development of many diseases, such as hormonedependent breast cancer (HDBC) [1]. One approach for treatment of the HDBC involves inhibitors of enzymes responsible for the biosynthesis of estrogens in peripheral tissues, e.g., steroid sulfatase (STS) [1]. The STS catalyses the hydrolysis reaction of steroid sulphates to their active forms and therefore plays a crucial role in the formation of biologically active hormones. The STS hydrolyses, among other, estrone sulfate (E1S) and dehydroepiandrosteronesulfate (DHEAS) into estrone (E1) and dehydroepiandrosterone (DHEA), respectively. The detailed studies have shown that E1 and DHEA can act as precursors for the formation of the estrogenic steroids estradiol (E2) and androstenediol (Adiol) [2]. Furthermore, the wide distribution of the STS in various tissues indicates that the STS enzyme is involved in numerous physiological and pathological conditions [3]. 
Because the activity of the STS may cause estrogenic stimulation of HDBC, research work focused on the design and synthesis of new and more effective agents that inhibit the STS enzyme is of particular importance and provides a major challenge for modern medicinal chemistry. In order to avoid the adverse estrogenic effects, in the recent years, there has been intensive research toward finding novel inhibitors based on non-steroidal cores (including coumarin derivatives). The first potent inhibitor based on the coumarin scaffold with significantly reduced estrogenic properties was 4-methyl-coumarin-7-O-sulfamate (1), Fig. 1 (COUMATE), which exhibited good activity with an $\mathrm{IC}_{50}$ value of $380 \mathrm{nM}$ when evaluated against placental microsomes [4]. Further modification of its structure led to a wide range of more potent compounds based on tricyclic coumarin derivatives containing sulfamate moiety that mimic the $\mathrm{ABC}$ rings of the natural substrate, e.g., 667COUMATE (2), Fig. 1 (currently in clinical trials) [5]. One of the strategies employed for generating a lead STS inhibitor involved replacement of the sulphate group of the natural enzyme substrate with surrogates or mimics other than sulfamate moiety, such as phosphates or thiophosphates [6]. Recently, Demkowicz et al. synthesized new phosphate and thiophosphate esters of tricyclic coumarin derivatives as potent STS inhibitors [7-9]. The most active compound, bis-(6oxo-7,8,9,10-tetrahydro-6H-benzo[c]chromen-3-yl) hydrogenthiophosphate (3) (Fig. 1) demonstrated the greatest inhibitory effect, with $\mathrm{IC}_{50}$ values of $860 \mathrm{nM}$ in enzymatic assay. Furthermore, the strategy based on introducing of fluorine atoms into the structure of coumarin sulfamate derivatives was examined. In 2016, the same research group synthesized a series of fluorinated 3-phenylcoumarin-7- $O$-sulfamates [10]. The most active compounds designing so far- 3-(3,4difluorophenyl)-coumarin-7-O-sulfamate (4), Fig. 1 and 3-(3,4,5-trifluorophenyl)-coumarin-7- $O$-sulfamate (5), Fig. 1, inhibited STS activity both with $\mathrm{IC}_{50}$ values of $270 \mathrm{nM}$.

The described above process of development of new steroid sulfatase inhibitors is expensive, time consuming, and

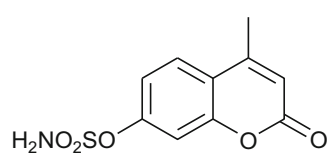

1

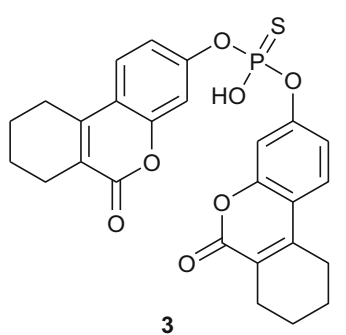

Fig. 1 Chemical structures of the STS inhibitors (1-5) requires collaboration of experts from different disciplines, such as: biology, chemistry, biochemistry, pharmacology, etc. However, according to recently recommended ideas in designing new drugs [11], this challenging process could be supported by a computational chemistry $[12,13]$. The computer-aided drug design (CADD) is a valuable and promising tool [14], especially in the contexts of the rational drug discovery. Recently, the world's major pharmaceutical and biotechnology companies more frequently follow the effectiveness strategies that allow reducing the costly failure of pharmaceutical candidates in clinical trials by applying different types of the computational techniques [12].

The computational methods employed in drug discovery can be classified into two main approaches: ligand-based drug design (LBDD) and structure-based drug design (SBDD) [15-17]. The first approach is applicable in the absence of information regarding the $3 \mathrm{D}$ structure of target molecules [16]. In this case, the quantitative-structure activity relationships (QSAR) [18] and pharmacophore modelling [19] could be applied. The presence of experimentally determined structure of target molecule would allow following the second type of methodology. This includes the molecular docking (MD) [20], QM-Polarized Ligand Dicking (QPLD) [21] or/and the three-dimensional quantitative-structure activity relationships (3D QSAR) [22, 23].

In the presented work, the application of the SBDD approach for the rational designing of new steroid sulfatase inhibitors is verifying. Due to the fact that the initial step both in molecular docking and 3D QSAR modelling involves the geometry optimization of the STS inhibitors, the main goal of our study (the first step according to application of SBDD) is to: (i) compare the geometries of the STS inhibitor structures after the optimization with methods differ in theory level; (ii) evaluate the impact of the geometry optimization on the values of descriptors coded chemical structure of STS inhibitors, and then, (iii) verify if the method applied to optimize the structures influence their free binding energy with the STS protein.

\section{Methodology}

\section{Comparison of the STS-inhibitors' geometries obtained at different theory level}

The molecular models of the 22 STS inhibitors presented in this study were built with the use of Gauss View [23] software. Chemical structures of the inhibitors were provided in Table 1. Then, the geometry of the compounds was optimized in the vacuum by two different methods: (i) the molecular mechanics (MM) using the MM+ force field and the Polak-Ribiere conjugate gradient algorithm terminating at the gradient of $0.05 \mathrm{kcal} \mathrm{mol}^{-1} \AA^{-1}$. The calculation was carried out with 
Table 1 Chemical structures of the STS inhibitors [6-10]

\begin{tabular}{|c|c|c|c|}
\hline ID & STS inhibitor & ID & STS inhibitor \\
\hline $\mathbf{a}$ & $\begin{array}{l}\text { 3-pentafluorophenylcoumarin-7-O- } \\
\text { sulfamate }\end{array}$ & 1 & $\begin{array}{l}\text { 6-oxo-7,8,9,10-tetrahydro-6H-benzo[c]chromen-3-yl } \\
\text { methylchlorothiophosphate }\end{array}$ \\
\hline b & $\begin{array}{l}\text { 3-(3,4-difluorophenyl)coumarin-7-O- } \\
\text { sulfamate }\end{array}$ & $\mathbf{m}$ & $\begin{array}{l}\text { 6-oxo-6,7,8,9,10,11-hexahydro- } \\
\text { cyclohepta[c]chromen-3-yl } \\
\text { methylchlorothiophosphate }\end{array}$ \\
\hline c & $\begin{array}{l}\text { 3-(3,4,5-trifluorophenyl)coumarin-7-O- } \\
\text { sulfamate }\end{array}$ & $\mathbf{n}$ & $\begin{array}{l}\text { 4-(2-Tridecanoylamino-ethyl)- } \\
\text { phenyldihydrogenphosphate }\end{array}$ \\
\hline d & $\begin{array}{l}\text { 3-[4-fluoro-3- } \\
\text { (trifluoromethyl)phenyl]coumarin-7-O- } \\
\text { sulfamate }\end{array}$ & o & bis-(6-oxo-7,8,9,10-tetrahydro-6H- \\
\hline e & $\begin{array}{c}\text { 3-[4-(trifluoromethoxy)phenyl]coumarin-7- } \\
\mathrm{O} \text {-sulfamate }\end{array}$ & $\mathbf{p}$ & $\begin{array}{l}\text { bis-(6-oxo-6,7,8,9,10,11-hexahydro- } \\
\text { cyclohepta[c]chromen-3-yl)-thiophosphoroamidate }\end{array}$ \\
\hline f & $\begin{array}{c}3-[2,5- \\
\text { Bis(trifluoromethyl)phenyl]coumarin-7-O- } \\
\text { sulfamate }\end{array}$ & $\mathbf{q}$ & $\begin{array}{l}\text { bis-(6-oxo-7,8,9,10-tetrahydro-6H- } \\
\text { benzo[c]chromen-3-yl)-hydrogenthiophosphate }\end{array}$ \\
\hline g & $\begin{array}{l}\text { 3-(4-phenylacetylamino-phenyl)-coumarin- } \\
\text { 7-O-sulfamate }\end{array}$ & $\mathbf{r}$ & $\begin{array}{l}\text { bis-(6-oxo-6,7,8,9,10,11-hexahydro- } \\
\text { cyclohepta[c]chromen-3-yl)-hydrogenthiophosphate }\end{array}$ \\
\hline h & $\begin{array}{l}\text { phenyl]-coumarin-7-O-sulfamate } \\
\text { 3-[4-(2-pentafluorophenyl-acetylamino)- }\end{array}$ & $\mathbf{s}$ & $\begin{array}{l}\text { 6-oxo-5,7,8,9,10,11-hexahydro-6H-cyclohepta[c] } \\
\text { quinolin-3-yl dihydrogenphosphate }\end{array}$ \\
\hline $\mathbf{i}$ & $\begin{array}{l}\text { coumarin-7-O-sulfamate } \\
\text { 3-(4-pentafluorobenzoylamino-phenyl)- }\end{array}$ & t & di(biphenyl-4-yl)-chlorothiophosphate \\
\hline $\mathbf{j}$ & benzo[c]chromen-3-yl dihydrogenphosphate & u & di(biphenyl-4-yl)- methylthiophosphate \\
\hline $\mathbf{k}$ & $\begin{array}{l}\text { 6-oxo-6,7,8,9,10,11-hexahydro- } \\
\text { cyclohepta[c]chromen-3-yl } \\
\text { dihydrogenphosphate }\end{array}$ & $\mathbf{v}$ & di(biphenyl-4-yl)-thiophosphoroamidate \\
\hline
\end{tabular}


the HyperChem [25] software and quantum mechanics (QM) using (ii) semi-empirical PM7 level was performed with the MOPAC 2012 [26] software; and (iii) Density Functional Theory (DFT) with Becke's Three Parameter Hybrid Method with the LYP (Lee-Yang-Parr) correlation functional (B3LYP) $[27,28]$ was applied as an ab initio algorithm. The $6-31++G^{*}[29,30]$ Pople's style, one-electron basis set was utilized. The DFT (B3LYP) calculations were performed with Gaussian 09 software> [31].

After geometry optimization we performed a Wilcoxon's tests for examining if the selected optimization methods influence the average bond lengths of the studied STS inhibitors. Subsequently, in order to obtain a deeper insight into the geometry optimization of selected compounds we compared individually their bond lengths, angles and dihedral angles at used data obtained in all calculations' levels $(\mathrm{MM}+\mathrm{PM} 7$, DFT B3LYP/6-31++G*).

\section{The influence of the geometry optimization on the values of descriptors coded the chemical structure of the STS inhibitors}

For compounds optimized at different theory level we calculated the 3D so-called molecular descriptors (840 descriptors), which describe the three dimensional structure of a particular compound. The descriptors were calculated with Dragon (version 6.0) software [32]. Then, we performed a series of statistical calculation (Wilcoxon's tests) for examining if the selected optimization methods influence on the descriptors' values. In this way, we were able to establish if the differences between value descriptors were significant.

\section{The influence of the geometry optimization on the free binding energy of steroid sulfatase inhibitors with STS - molecular docking}

\section{Protein preparation}

The X-ray structure of the human steroid sulfatase (STS) was taken from the Protein Databank (PDB ID: 1P49) and prepared for docking using the following procedure: (i) the catalytic amino acid FGly75 (formylglycine) was converted to the gem-diol form using the Discovery Studio visualizer (http://accelrys.com/products/collaborative-science/bioviadiscovery-studio/visualization.html), (ii) the waters of crystallization were removed from the structure, (iii) polar hydrogen atoms were added to the protein, and (iv) gasteiger charges were added to each atom and the non-polar hydrogen atoms were merged to the protein structure employing Autodock Tools 1.5.6. [33].

The distance between donor and acceptor atoms that form a hydrogen bond was defined as $1.9 \AA$ with a tolerance of $0.5 \AA$, and the acceptor-hydrogen-donor angle was not less than $120^{\circ}$. The structure was then saved in PDB file format for PatchDock docking and in PDBQT file format for docking studies in Autodock Vina 1.1.2 software [33], (http://autodock.scripps.edu/resources/references).

\section{Ligand preparation}

The STS inhibitors presented in Table 1 (optimized at above describe three levels of theory: (i) MM+, (ii) PM7 and (iii) DFT B3LYP $6-31++G^{*}$ ) were analysed in order to select compounds structurally similar to 667-COUMATE that was thoroughly studied according to mode of inhibitor binding to STS [34]. Hierarchical cluster analysis (HCA) with Euclidian distances and Ward's method of linkage [34] was applied. Selected inhibitors were saved as PDB file format for input to PatchDock software [35]. To carry out docking study in AutodockVina 1.1.2 software, all the ligand structures were saved also in PDBQT file format in Autodock Tools 1.5.6. [33].

\section{Docking studies}

AutoDock The docking of the optimized inhibitors into the prepared rigid structure of the human steroid sulfatase protein was performed using the Autodock Vina 1.1.2 software [33]. For all the docking studies, a grid box size of $30 \AA \times 30 \AA \times 30 \AA$ centred on the $\mathrm{C} \beta$ atom of the amino acid FGly 75 was used. The centre of the box was set at ligand centre and grid energy calculations were carried out. For the AutoDock docking calculation, default parameters were used and the 20 docked conformations were generated for each compound. The energy calculations were performed employing the genetic algorithms (GAs). All dockings were taken into 2.5 million energy evaluations for each of the test molecules. In order to verify the reproducibility and validation of the docking calculations, 667-COUMATE was submitted as reference molecule for one-ligand run calculation. The active pocket consisted of identical amino acid residues for 667COUMATE from literature [7] suggesting that this method is valid enough to be used for docking studies of other test ligands. Docking of all ligands to protein was performed using AutoDock following the same protocol used for reference compound. Docked ligand conformations were analysed in terms of energy, hydrogen bonding, and hydrophobic interaction between ligand and receptor protein human STS.

PatchDock The PatchDock [35] is geometry-based molecular docking open source web software designed to find docking transformations facilitating excellent molecular shape complementarity. Such transformations, when applied, induce both wide interface areas and small amounts of steric clashes which ensured to include numerous matched local features of the docked molecules that have complementary characteristics. 
Therefore, we decided to use PatchDock docking to compare the results of AutoDock and make the study using the approach proved to be more accurate one.

As it is a web-based software, both the prepared rigid protein and ligands saved in the PDB format were uploaded. Followed by root mean square deviation (RMSD) value set to 1.5 clustering is applied to the candidate solutions to discard redundant solutions. Each candidate transformation is further evaluated by a scoring function that considers both geometric fit and atomic desolvation energy [35]. The geometric score, the desolvation energy, the interface area size and the actual rigid transformation of the solution were provided in the output file to judge the best possible docked conformations. The main reason behind PatchDock's high efficiency is its fast transformational search, which is driven by local feature matching rather than brute force searching of the sixdimensional transformation space.

Finally, we compared the free binding energy from the docking calculations with the experimental values. This was performed in order to investigate the influence of the geometry optimization method on the free binding energy of the ligand and the STS active site.

\section{Results and discussion}

\section{Comparison of geometries of STS-inhibitors obtained at different theory level}

The geometries of the 22 steroid sulfatase inhibitors (see Table 1) were optimized with application of two methods differing in theory level. We have applied: (i) the molecular mechanics using the MM+ force field and the Polak-Ribiere conjugate gradient algorithm terminating at the gradient of $0.05 \mathrm{kcal} \mathrm{mol}^{-1} \AA^{-1}$; (ii) the quantum-mechanics using semi-empirical PM7 level; and DFT B3LYP with the 6$31++\mathrm{G}^{*}$ basis set. The application of DFT B3LYP $6-31++$ $\mathrm{G}^{*}$ method for coumarin derivatives was previously verified [36, 37].

In order to compare the obtained geometries, we have taken into account three parameters describing geometry: (i) bond lengths; (ii) angles; and (iii) dihedral angles [36-38]. First, we have considered bond lengths. Thus, we have calculated average bond lengths (sum of bond lengths divided by number of bonds) for each STS-inhibitor. Then, we performed a series of the statistical Wilcoxon's tests (at 1\% level of confident), comparing the average bond lengths obtained for the whole set of structures optimized at different calculation levels. According to the obtained results, Table 2, it can be noticed that there are no statistically significant differences related to average bond lengths computed with applied methods.

In the next step we compared the bond lengths, angles and dihedral angles individually for each STS-inhibitor. Figure 2
Table 2 Results of Wilcoxon test for comparison of average bond lengths of each STS-inhibitor

\begin{tabular}{lll}
\hline & $\mathrm{T}_{\min }$ & $\mathrm{T}_{\alpha=0,001}$ \\
\hline MM+ vs. PM7 & 35 & 30 \\
MM+ vs. DFT B3LYP 6-31++G* & 56 & \\
PM7 vs. DFT B3LYP 6-31++G* & 32 & \\
\hline
\end{tabular}

presented the result for one structure (j, Fig. 2A). For the results related to other selected studied inhibitors please refer to the Supplementary Info (Table S1). The comparison of particular bond lengths for geometries computed at different theory levels (Fig. 2B) allows us to confirm results obtained using approach with average bond lengths: there are negligible differences between the bond length; the most significant are related to structures optimized with the $\mathrm{MM}+$ method in comparison with the PM7 and DFT B3LYP 6-31++G* ones. Similar results were noticed comparing angles between particular atoms in STS-inhibitors' structures (Fig. 2C). Simultaneously, the highest impact of geometry optimization was observed in the case of the dihedral angles measured between planes containing central atom that can be rotated. This differences were the most significant for compounds optimized with the MM+ method (Fig. 2D), which is reasonable considering the theory level that this technique is based on. The region of the system where the optimization process takes place, for example bond breaking and formation is larger in MM approach than in QM ones [39]. This means that in case of the MM method more significant changes in the structures are possible. Analysis of the change in energy of the compounds as a function of angle of torsion about the O12-P13 bond indicates that in this approach various conformers are created.

Due to the fact that there are differences in geometries suggesting that optimization method influences the type of conformer creation, we decided to evaluate the energies of structures obtained in each optimization. Therefore, we have calculated for each structure its total energy.

The total energies (TEs), calculated with the DFT B3LYP/ 6-31++G* method for optimized geometries are similar regardless of applied method of optimization, Table 3. This indicates that all the obtained structures, even though there are differences in geometries, are on the same energy level. It confirms that these differences are related to differences in conformer creation [40]. Additionally, to verify if this energy is the most favourable one, the application of two approaches applied so far separately has been used together. Such approach is recommended in order to gain structure in its global minimum considering its total energy [41]. Therefore, geometries of the selected STS-inhibitors optimized with the MM+ technique were once more optimized with the DFT B3LYP/6 $31++\mathrm{G}^{*}$ approach. To obtain the representative subset of 
a

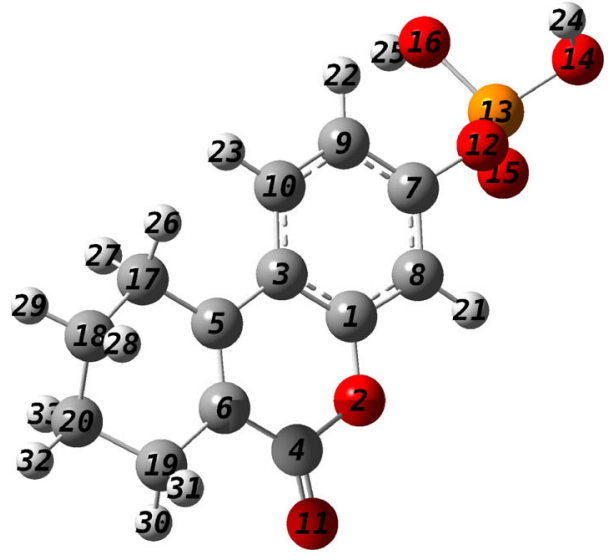

b

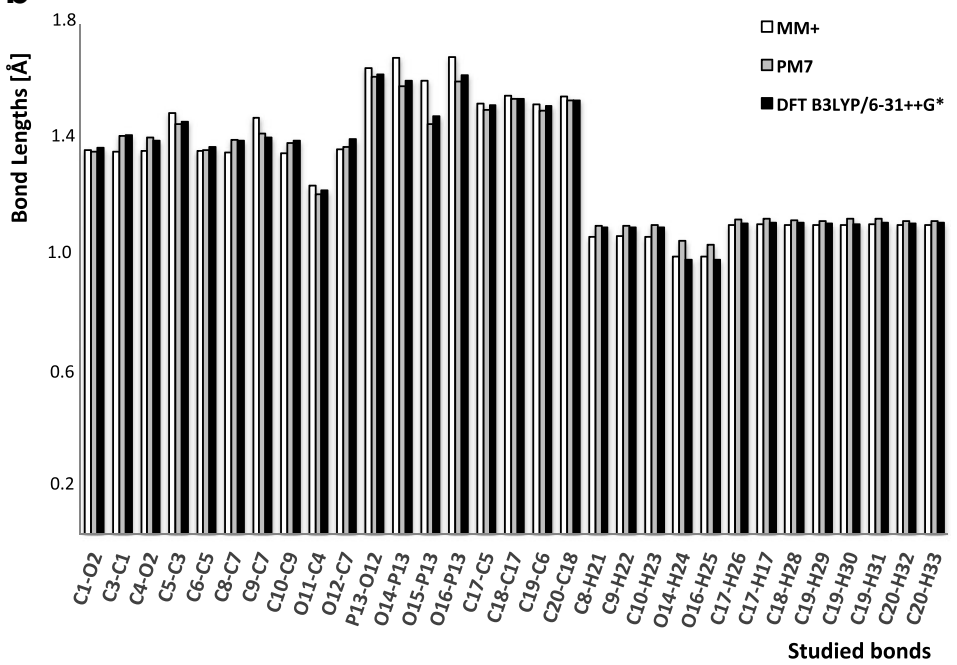

C

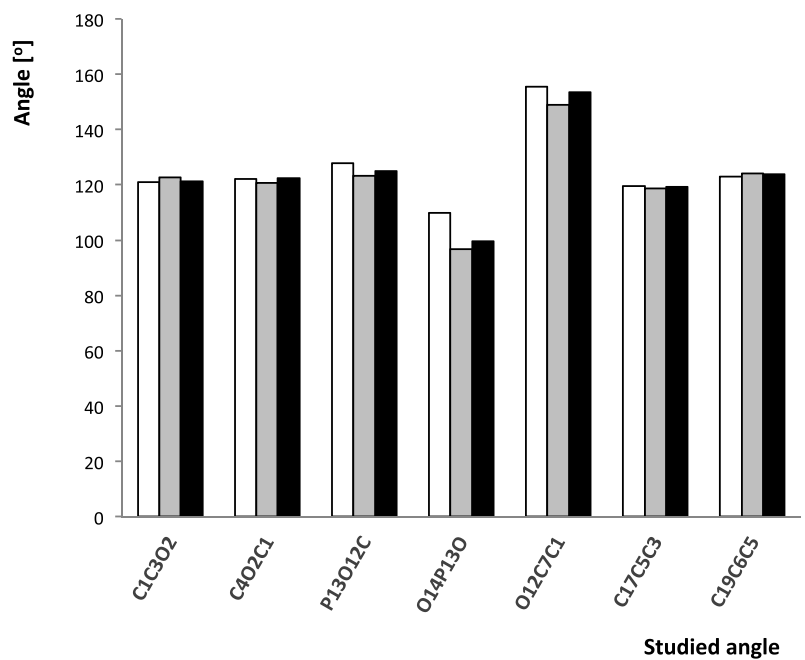

d

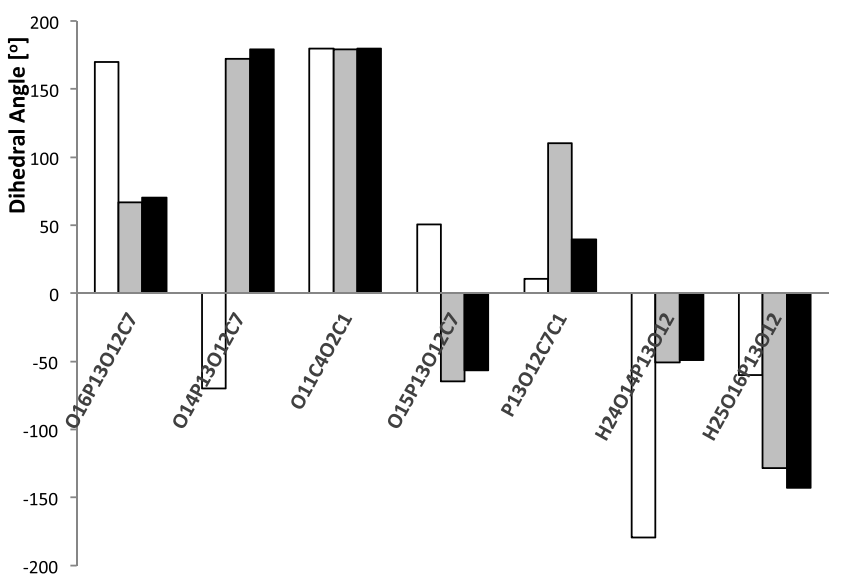

Fig. 2 a Chemical structure of 6-oxo-7,8,9,10-tetrahydro-6H-benzo[c]chromen-3-yl dihydrogenphosphate; $\mathbf{b}$ its bond lengths, $\mathbf{c}$ angles and $\mathbf{d}$ dihedral angles at various calculations' levels

inhibitors, we selected compounds differing in their chemical structures. We obtained the following energies: $\mathrm{TE}(\mathbf{f})=-5.464 * 10^{6} \mathrm{kJmol}^{-1}, \mathrm{TE}(\mathbf{j})=-3.403 * 10^{6} \mathrm{kJmol}^{-1}$, $\mathrm{TE}(\mathbf{m})=-5.466 * 10^{6} \mathrm{kJmol}^{-1}, \mathrm{TE}(\mathbf{n})=-4.186 * 10^{6} \mathrm{kJmol}^{-1}$, $\mathrm{TE}(\mathbf{s})=-3.454 * 10^{6} \mathrm{kJmol}^{-1}$. Energies were similar to the ones computed in previously applied methods. This confirms that regardless of the applied method structures representing their most favourable energy are obtained.

Moreover, we have compared also the geometry of compounds optimized with MM+/DFT B3LYP/6-31++G* approach with the previously applied ones. According to the obtained results, Figures S1-S3, one can notice that the choice of the applied method poses the highest impact on the dihedral angles between planes including the central atom that can be rotated (e.g. dihedral angle created by atoms of functional group of compound $\mathbf{j}$ ). Taking into account that the total energies computed for STS-inhibitors are similar, as we have proven above, one can conclude that the method of optimization has impact on the type of molecular conformer creation $[36,42]$. The application of the PM7 and the DTF B3LYP/6$31++\mathrm{G}^{*}$ approach allow obtaning the same conformer. Application of the method based on molecular mechanics forces the creation of another type of conformer.

The influence of the geometry optimization on the values of the descriptor coded chemical structure of the STS inhibitors

In order to obtain deeper insight into the geometry optimization results we have performed further analysis that tested the influence of the chosen optimization method $(\mathrm{MM}+, \mathrm{PM} 7$, DFT B3LYP/6-31++G*) on the molecular descriptor values. In the comparison study we chose descriptors, which might be affected by the 3D structure of the molecule. We selected 
Table 3 Comparison of total energy calculated with the DFT B3LYP 6-31++ G* method for geometries obtained at different theory levels

\begin{tabular}{|c|c|c|c|c|}
\hline \multirow[b]{2}{*}{ Method of geometry optimization } & & \multicolumn{3}{|l|}{ Energy $^{\mathrm{a}}$} \\
\hline & & $\mathrm{MM+}$ & $\mathrm{B} 3 \mathrm{LYP} / 6-31++\mathrm{G}^{*}$ & PM7 \\
\hline \multirow[t]{22}{*}{ Total energy $\times 10^{-6}\left[\mathrm{~kJ} \mathrm{~mol}^{-1}\right]$} & a & -4.997 & -4.997 & -4.997 \\
\hline & $\mathrm{b}$ & -4.216 & -4.216 & -4.216 \\
\hline & $\mathrm{c}$ & -4.476 & -4.476 & -4.476 \\
\hline & $\mathrm{d}$ & -4.840 & -4.840 & -4.840 \\
\hline & $\mathrm{e}$ & -4.777 & -4.777 & -4.777 \\
\hline & $\mathrm{f}$ & -5.464 & -5.465 & -5.465 \\
\hline & $\mathrm{g}$ & -4.847 & -4.848 & -4.848 \\
\hline & $\mathrm{h}$ & -6.150 & -6.150 & -6.150 \\
\hline & $\mathrm{i}$ & -6.047 & -6.047 & -6.047 \\
\hline & $\mathrm{j}$ & -3.403 & -3.403 & -3.403 \\
\hline & $\mathrm{k}$ & -3.506 & -3.506 & -3.506 \\
\hline & 1 & -5.363 & -5.363 & -5.363 \\
\hline & $\mathrm{m}$ & -5.466 & -5.466 & -5.466 \\
\hline & $\mathrm{n}$ & -4.186 & -4.186 & -4.186 \\
\hline & o & -5.910 & -5.910 & -5.910 \\
\hline & $\mathrm{p}$ & -6.116 & -6.117 & -6.116 \\
\hline & $\mathrm{q}$ & -5.962 & -5.962 & -5.962 \\
\hline & $\mathrm{r}$ & -6.169 & -6.169 & -6.169 \\
\hline & $\mathrm{s}$ & -3.454 & -3.454 & -3.454 \\
\hline & $\mathrm{t}$ & -5.975 & -5.975 & -5.975 \\
\hline & $\mathrm{u}$ & -5.069 & -5.069 & -5.069 \\
\hline & $\mathrm{v}$ & -4.914 & -4.914 & -4.914 \\
\hline
\end{tabular}

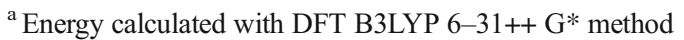

groups of 3D descriptors such as: Radial Density Function descriptors (RDF), 3D-MOlecule Representation of Structures based on Electron diffraction (3D-MoRSE), Weighted Holistic Invariant Molecular descriptors (WHIM), GEometry, Topology and Atom-Weight Assembly descriptors (GETAWAY), Molecular properties and Drug-like indices. Then, each group of selected descriptors was divided into smaller sub-groups connected to their weighting scheme (unweighted (u), weighted by mass (m), by van der Waals volume (v), by Sanderson electronegativity (e), by polarizability (p), by ionization potential (i), and by I-state (s). Afterwards, we have compared descriptor values for each STS inhibitor with its analogue from the sub-group optimized with different optimization methods. We have applied a series of statistical Wilcoxon's tests (at $1 \%$ level of confidence). The number of performed test was equal to the number of the descriptor's sub-group for each STS inhibitor.

According to the results presented in Fig. 3 we have deduced, that there are groups of descriptors, which are sensitive on the geometry optimization method. The RDF descriptors (which describe the distance distribution in the molecule) exhibited significant different values for several STS inhibitor descriptors comparing the structure optimized with DFT
B3LYP/6-31++G* and MM+ (panel B, sub-groups 1-7). This suggest that similar values of the RDF descriptors were calculated only for the STS inhibitor's structure optimized with PM7 and MM+ (panel C, sub-group 1-7), and PM7 and $\mathrm{B} 3 \mathrm{LYP} / 6-31++\mathrm{G}^{*}$ (panel A, sub-group 1-7). In the case of the 3D-MoRSE descriptors (group which is based on the electron diffraction, where the weighted scheme could be used to identify the presence of specific molecular fragments) only for few compounds the differences in descriptor values (obtained after optimization by all three methods) are significant. WHIM weighted and unweighted descriptors (which deliver information about the molecule's 3D structure, regarding molecular size, shape, symmetry and atom distribution) constitute the class where the influence of the different optimization methods can be negligible (with only few exceptions from this trend). On the other hand, in the case of WHIM total descriptors one can notice that in all the compared optimization methods (panels A-C, sub-group 22) the differences in the descriptor values are significant. This is most noticeable in comparison of the PM7 and MM+ methods (panel C), where 19 of the 22 compounds have statistically different descriptor values. Next, the group of GETAWAY descriptors seems to be the most affected by the geometry optimization methods. The 


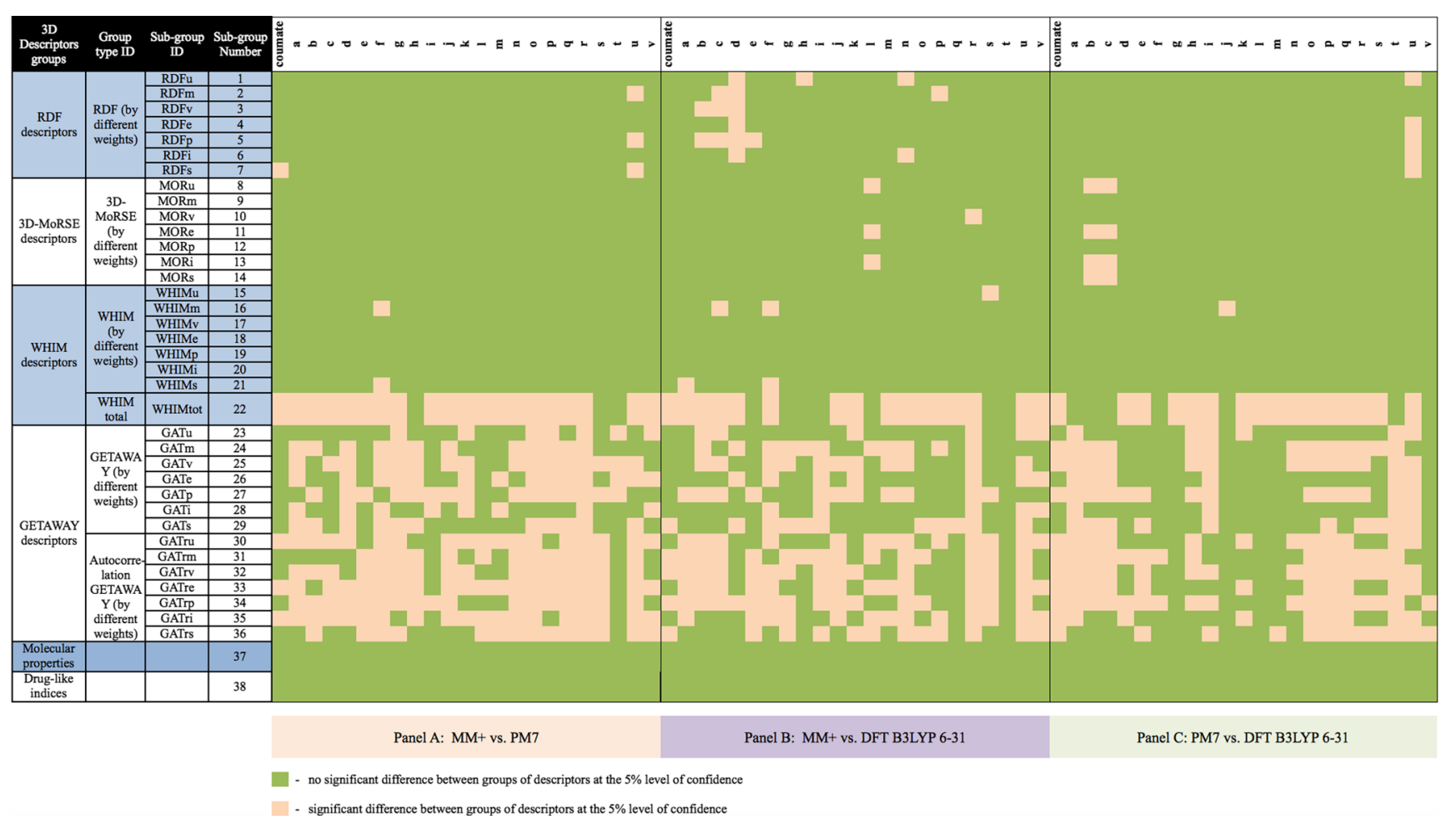

Fig. 3 Results of Wilcoxon's test for 3D descriptors groups

GATEWAYs descriptors deliver on one hand the information about the particular atoms' influence on the shape of the whole molecule and their ability to "interact" with each other, whereas on the other hand information about spatial distance between pairs of atoms. There are significant statistical differences in descriptor values in every optimization method for the majority of the studied compounds. Only the sub-classes of unweighted GATEWAYs and GATEWAYs weighted by ionization potential descriptors are rather not susceptible to the kind of optimization methods - with few expectations. What is more, the group of autocorrelation GATEWAY's seems to be more sensitive for the molecule's optimization method (panels A-C, sub-groups 23-27) than the weighted GATEWAYs. Finally, according to the results, the Molecular properties (set of heterogeneous molecular descriptors describing physico-chemical and biological properties) and Drug-like index descriptor values for all STS inhibitors do not significantly differ after all the applied optimization method (panels A-C, sub-groups 37-38).

Summarizing, we have proved that the selection of the geometry optimization method did not affect the optimal STS inhibitor coordinates, and hence the values of molecular descriptors which describe the 3D structure of the molecule. This trend was noticed in most classes of calculated 3D descriptors. In the next step, in order to verify, if the optimization methods influence the binding mode of the STS inhibitor to the active site we performed further analysis.
The influence of the geometry optimization on the free binding energy of the steroid sulfatase inhibitors with STS - molecular docking

To compare the influence of the inhibitors' geometries on their free binding energy with the steroid sulfatase we have selected inhibitors structurally similar to 667-COUMATE that was proven to bind with the binding pocket of the protein [34]. The selection was performed with the application of HCA with Euclidian distances and Ward's method of linkage [43]. The similarities were analysed in space of the topological descriptors.

According to Fig. 4 we selected five compounds to assess the influence of the STS-inhibitors' geometries on their binding with the protein. Selected compounds are as follows: $\mathbf{j}, \mathbf{k}$, $\mathbf{l}, \mathbf{m}$ and $\mathbf{s}$ (Table 1.). All the selected derivatives and the reference compound (667-COUMATE) were optimized separately with application of the three described in the Methodology section: (i) MM+; (ii) PM7; and (iii) DFT B3LYP/6-31++G*. In the case of protein, for all the docking studies, a grid box size of $30 \AA \times 30 \AA \times 30 \AA$ centred on the $\mathrm{C} \beta$ atom of the amino acid FGly 75 was used. The centre of the box was set at the ligand centre and grid energy calculations were carried out. The binding energies were computed by means of molecular docking with application of two programs: AutoDock and PatchDock. One has to understand that though the basic approach of all docking software is the same the algorithm behind the docking technique varies from 
Fig. 4 Hierarchical Cluster Analysis of STS-inhibitors in the space of topological descriptors

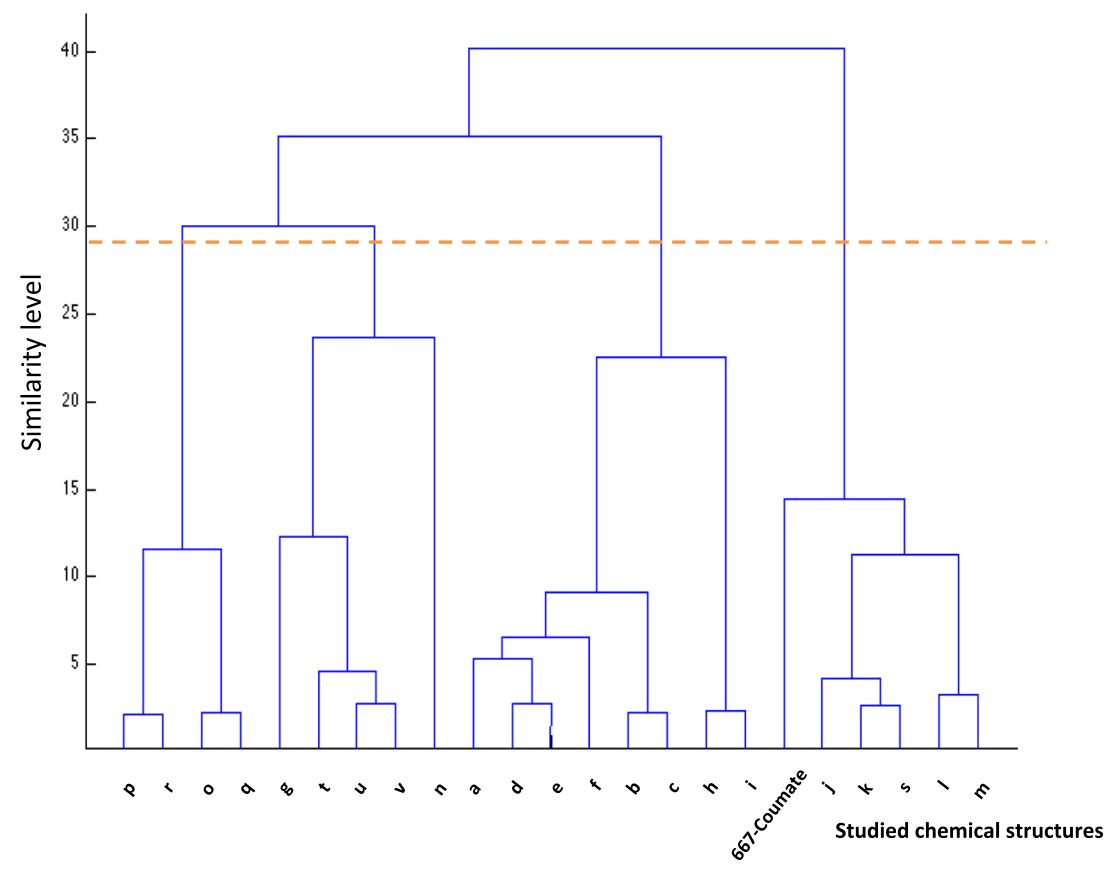

software to software. Selection of these two software packages is reasonable because we intend first, to validate the docking studies and second, to understand the interaction pattern of the studied molecules with the target receptor.

Meticulous analyses of the ligand-receptor interactions were carried out, and final coordinates of the ligand and receptor were saved. The Discovery Studio visualizer (http://accelrys.com/products/collaborative-science/bioviadiscovery-studio/visualization.html) was employed for display of the receptor with the ligand-binding site. The docking of the inhibitors of the human STS with the receptor (1P49) exhibited well-established bonds with one or more amino acids in the receptor active pocket. The active pocket consisted of amino acid residues as Leu74, Arg98, Gly100, Val101, Leu167, His290, His346, Lys368, Asn447, Val 486 and Phe488.

\section{AutoDock outcome}

Docking results for MM+ optimized geometries Docking studies revealed that the synthesized molecules optimized through the $\mathrm{MM}+$ method showed diverse free binding energy toward the target protein, ranging from -3.50 to $2.90 \mathrm{~kJ}^{*} \mathrm{~mol}^{-1}$. Molecule $\mathbf{j}$ is characterized by the least free binding energy of $-3.50 \mathrm{~kJ}^{*} \mathrm{~mol}^{-1}$ among the experimental compounds by fitting in the active protein sites making interactions with FGly75, Leu74, Arg98, Gly100, Lys368, Phe488 and Asn447. Figure 5 shows a putative enzyme-ligand complex before the recognized inactivation of the STS and the overlaid best conformations of the four derivatives $(\mathbf{j}, \mathbf{k}, \mathbf{l}$ and s). Interestingly, the designed STS inhibitors exhibited similar docked conformation matched with the reference sulfamate-based STS inhibitor (667-Coumate). For these compounds, the core structure of coumarin derivatives were oriented in the centre of the active site and underwent a nonpolar interaction with the side chains of the hydrophobic pocket formed by the Leu74, Arg98, Gly100, Val101, Leu167, His290, Lys368, and Phe488 residues. On the contrary, though compound $\mathbf{m}$ is surrounded with the same amino acid residues their orientation was completely opposite of the other experimental compounds along with COUMATE. Considering docked conformation, flexible side chain i.e. thiophosphate derivative of compound $\mathbf{m}$ is not able to provide any form of interactions with Leu74, Arg98, Gly100, Lys368 and Phe488 due to the alternate orientation which support higher binding energies comparing to compound $\mathbf{j}$ and the reference compound (Fig. 5).

Docking results for PM7 optimized geometries In silico studies revealed all the synthesized molecules showed moderate free toward the target receptor, ranging from -4.56 to $-1.03 \mathrm{~kJ} \mathrm{~mol}^{-1}$. The predicted free binding energy was more favourable in the case of molecule (j), which led to the lowest free binding energy of $-4.56 \mathrm{~kJ} \mathrm{~mol}^{-1}$ among the experimental compounds by fitting exactly in the active sites making interactions with Arg98, Phe488, Asn447 and Val486. Figure 6 displays a putative enzyme-ligand complex before the presumed inactivation of the STS and the superimposed best conformations of the three derivatives (j, $\mathbf{k}$ and $\mathbf{s}$ ). As shown in Fig. 6, the designed STS inhibitors exhibited the same docked conformation compared with the reference sulfamate-based STS inhibitor (667-Coumate). In this case, the skeleton of coumarin derivatives were oriented in the centre of the active site and underwent a non-polar interaction 


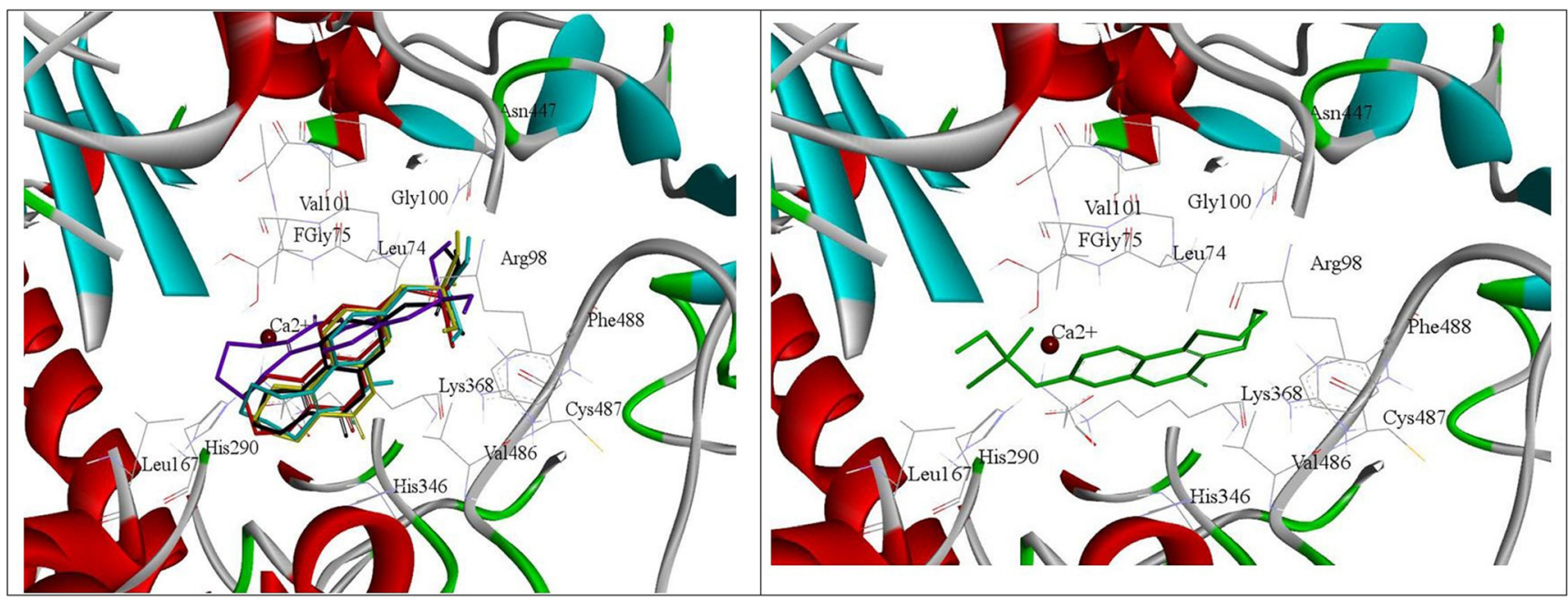

Fig. 5 Docked binding modes for the compounds $\mathbf{j}$ (Yellow), $\mathbf{k}$ (Purple), $\mathbf{I}$ (Red), $\mathbf{s}$ (Cyan) and COUMATE (black) in left and for the compound $\mathbf{m}$ (Green) in right for MM + method in AutoDock software

with the side chains of the hydrophobic pocket formed by the Leu74, Arg98, Gly100, Val101, Leu167, Val486, and Phe488 residues. On the contrary, though compounds like $\mathbf{I}$ and $\mathbf{m}$ surrounded with the same amino acid residues their orientation was completely opposite of the other experimental compounds as well as with the reference compound COUMATE. In this case, flexible side chain i.e. phosphate derivative cannot make any form of interactions with Arg98, Phe488, Asn447 and Val486 due to opposite orientation which support higher binding energies of compounds $\mathbf{I}$ and $\mathbf{m}$ comparing to compound $\mathbf{j}$ and the reference compound (Fig. 6). It is interesting to point out that the orientation of compound $\mathbf{l}$ for the other two optimization methods (MM+ and DFT approach) were completely opposite than the orientation but the orientation of compound $\mathbf{m}$ for all the three methods was the same.

Docking results for DFT B3LYP/6-31++G* optimized geometries Comparing the free binding energy of the docked conformation of the studied molecules, compound $\mathbf{j}$ is observed to have the least free binding energy of
$-3.96 \mathrm{~kJ} * \mathrm{~mol}^{-1}$ making interactions with Leu74, Arg98, Gly100, Lys368, Phe488 and Asn447. Figure 7 shows the best conformations of the four derivatives $\mathbf{j}, \mathbf{k}, \mathbf{l}$ and $\mathbf{s}$ exhibited comparable docked conformation with the reference sulfamate-based STS inhibitor 667-Coumate. Like the MM+ method, the scaffold of the coumarin derivatives was encircled with the side chains of the hydrophobic pocket formed by the Leu74, Arg98, Gly100, Val101, Leu167, Lys368, and Phe488 residues as well as by the polar amino acids like His290 and Asn447. In this case also, although the same amino acid residues were making the cavity for compound $\mathbf{m}$ its orientation was completely reversed than the other experimental compounds along with COUMATE. Analysing docked conformation, flexible side chain i.e. the thiophosphate derivative of compound $\mathbf{m}$ is not able to make any form of interactions with Leu74, Arg98, Gly100, Lys368 and Phe488 due to its opposite orientation which supports its bad binding energies comparing to the other compounds and the reference compound (Fig. 7).
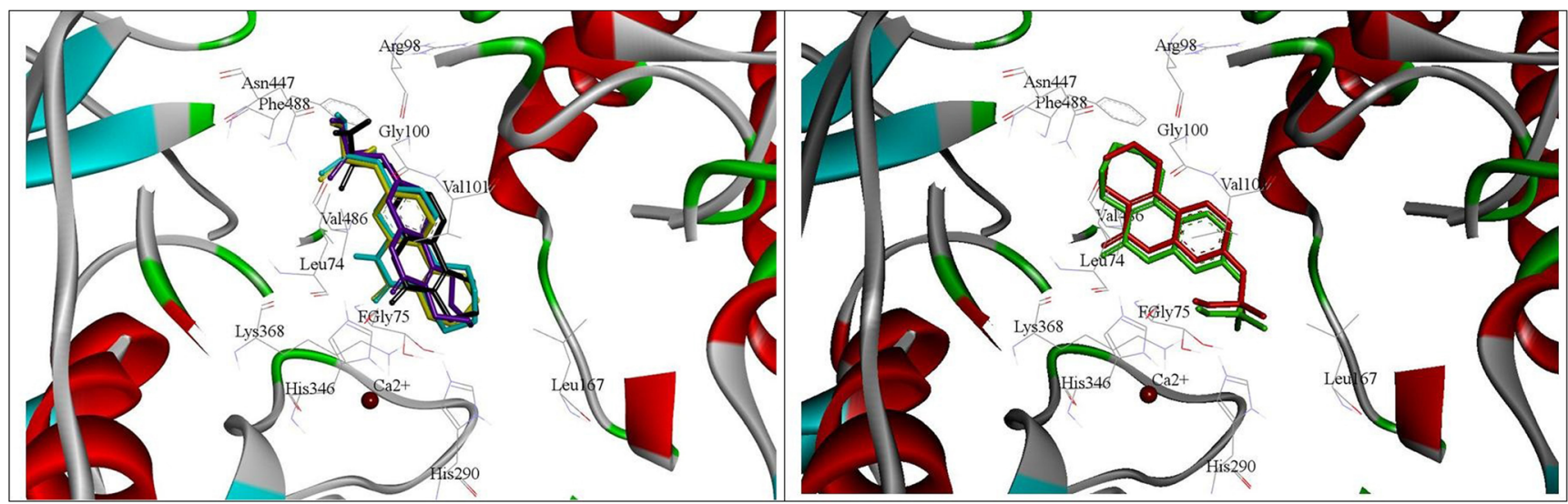

Fig. 6 Docked binding modes for the compounds $\mathbf{j}$ (Yellow), $\mathbf{k}$ (Purple), $\mathbf{s}$ (Cyan) and COUMATE (Black) in left and for the compounds $\mathbf{I}$ (Red) and $\mathbf{m}$ (Green) in right for PM7 method in AutoDock software 


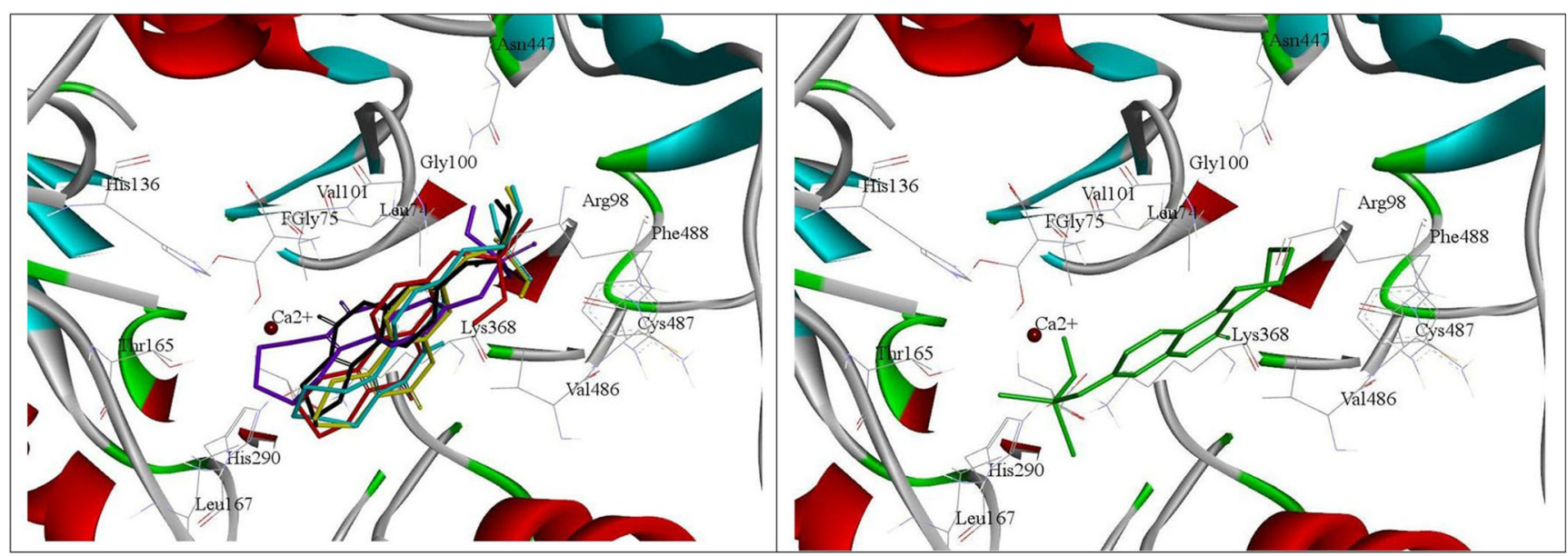

Fig. 7 Docked binding modes for the compounds $\mathbf{j}$ (Yellow), $\mathbf{k}$ (Purple), $\mathbf{I}$ (Red), $\mathbf{s}$ (Cyan) and COUMATE (black) in left and for the compound $\mathbf{m}$ (Green) in right for DFT B3LYP/6-31++G* method in AutoDock software

\section{PathDock outcome}

Docking results for MM+ optimized geometries Molecules optimized with the MM+ method led to values within the atomic contact energy range between -8.7 to $-6 \mathrm{kcal} \mathrm{mol}^{-1}$. The most potent compound $\mathbf{m}$ according to the biological assay showed the best atomic contact energy of $-8.7 \mathrm{kcal}^{*} \mathrm{~mol}^{-1}$. The active pocket for the docked conformation of the synthesized coumarin derivatives along with COUMATE were comprised of amino acid residues like Arg98, Val 101, Val177, Phe178, Thr180, Val486 and Phe488. Analysing the inhibitor enzyme interactions at the atomic level, it can be identified that the phosphate or phiophosphate moieties of the $\mathbf{j}$ and $\mathbf{I}$ compounds were directed toward the $\operatorname{Arg} 98$ residue and that their amine groups were able to establish hydrogen bonds (Fig. 8). This could favour the binding and may have a significant impact on the enzyme-ligand complex stability [7]. Interestingly, the reference compound had shown similar orientation in the active site pocket like compounds $\mathbf{j}$ and $\mathbf{l}$. On the contrary, in the case of compounds like $\mathbf{k}, \mathbf{m}$ and $\mathbf{s}$ (Fig. 8), the opposite arrangement of the phosphate or thiophosphate moieties was noticed. Interestingly, the reason behind the best free binding energy of compound m was the chance of making hydrogen bond more prominently with Thr484 than with the other two compounds $\mathbf{k}$ and $\mathbf{s}$ due to their slightly shifted orientations from Thr484.

Docking results for PM7 optimized geometries Synthesized derivatives optimized with PM7 method led to values within the atomic contact energy range between -9.6 to $-5.6 \mathrm{kcal} \mathrm{mol}^{-1}$. The most potent compound $\mathbf{m}$ according to the biological assay showed the best atomic contact energy of $-9.6 \mathrm{kcal} \mathrm{mol}^{-\mathbf{1}}$. The active pocket for the docked conformation of the synthesized coumarin derivatives consisted of amino acid residues as Arg98, Val101, Val177, Thr180, Gly181, His 290, Lys368, Thr484, His485, and Phe488. Analysing the inhibitor enzyme interactions at the atomic level, it can be identified that the phosphate or thiophosphate moieties of the $\mathbf{j}, \mathbf{k}, \mathbf{m}$ and $\mathbf{s}$ compounds were directed toward the Thr484 residue and that their $\mathrm{OH}$ groups are able to establish hydrogen bonds (Fig. 9). It is already proven that the identified hydrogen bond between the phosphate moieties and the Thr484 residue could favour the binding and may have a significant impact on the enzyme-ligand complex stability [7]. On the contrary, in the case of the compound I (Fig. 9), the opposite arrangement of the thiophosphate moiety to the hydroxyl group of Thr484 suggests that a thiophosphate group transfer may be crucial during the inactivation process.

Docking results for DFT B3LYP/6-31++G* optimized geometries Like in all other methods, compound $\mathbf{m}$ showed the best atomic contact energy of $-9.3 \mathrm{kcal} \mathrm{mol}^{-\mathbf{1}}$ justifying its experimental assay result. Docked conformation of all the synthesized compounds for the DFT method placed among the following amino acid residues: Arg98, Val101, Val177, Thr180, His290, Lys368, Thr484, His485, and Phe488. Docking results revealed that the phosphate or thiophosphate moieties of compounds $\mathbf{k}, \mathbf{m}$ and $\mathbf{s}$ were directed toward the $\mathrm{Thr} 484$ residue and that their $\mathrm{OH}$ groups are able to establish hydrogen bonds (Fig. 10). Again, for compounds $\mathbf{j}$ and $\mathbf{l}$ (Fig. 10), complete opposite arrangement of the phosphate or thiophosphate moieties to the hydroxyl group of Thr 484 suggests that a phosphate or thiophosphate group transfer may be crucial during the inactivation process. 


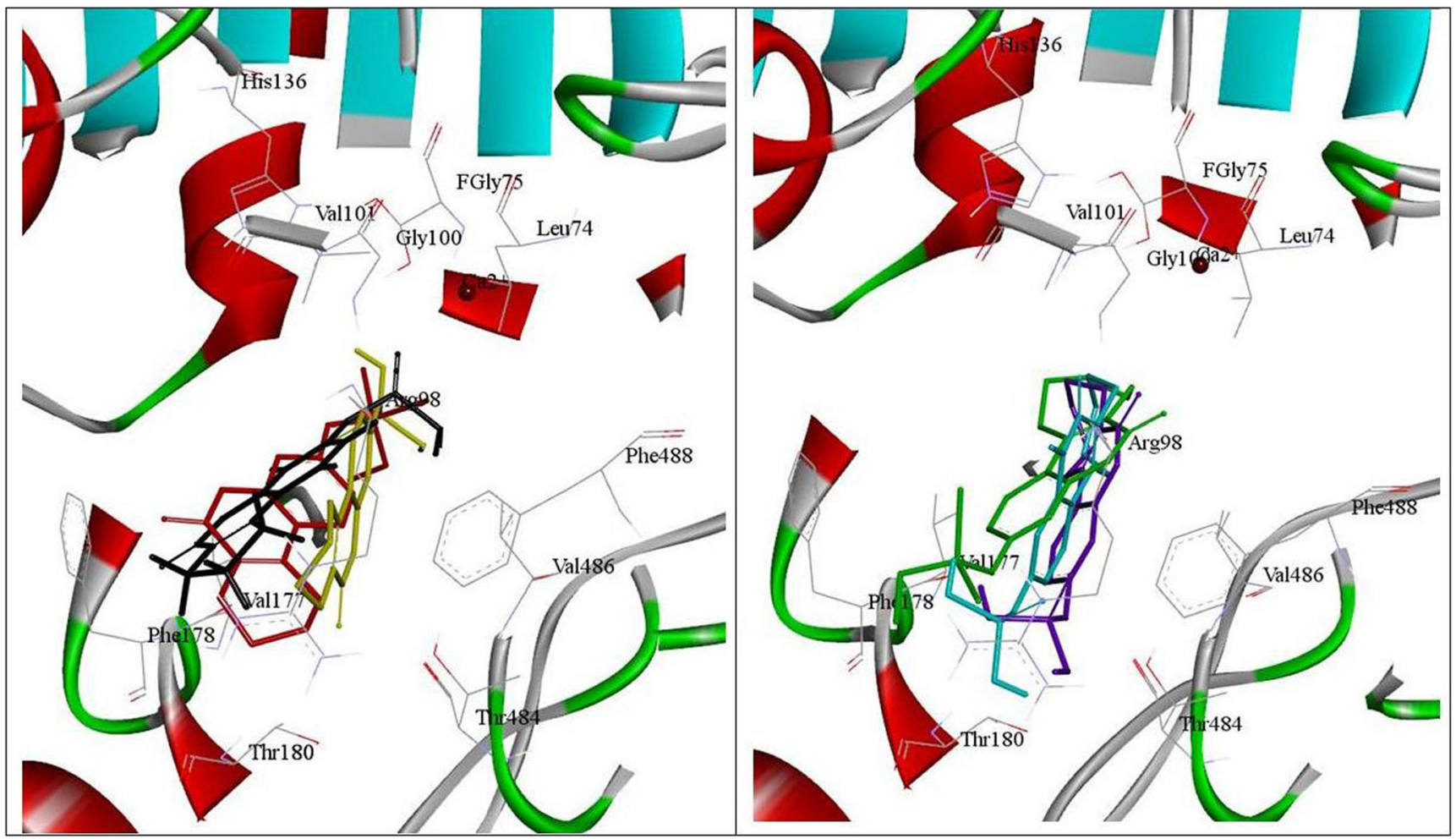

Fig. 8 Docked binding modes for the compounds $\mathbf{j}$ (Yellow), $\mathbf{l}$ (Red), and COUMATE (Black) in left and for the compound $\mathbf{k}$ (Purple), $\mathbf{m}$ (Green) $\mathbf{s}$ (Cyan) in right for MM+ method in PatchDock software

The free energy of binding of selected derivatives with STS was calculated by means of AutoDock and PatchDock and the results are presented in Table 4.

After obtaining the free binding energy for the STS inhibitors we compared these values with the experimental ones. In this case, we calculated the binding affinities according to the formula $\Delta \mathrm{G}=-\mathrm{RT} \ln \mathrm{K}_{\mathrm{i}}$, [44]. We adapted the working hypothesis introduced by Durdagi et al., Naik et al. and Conn et al. that assume that $\mathrm{K}_{\mathrm{i}}$ is equal to the $\mathrm{IC}_{50}[\mu \mathrm{M}]$ [45-47]. The residual values correspond to the binding energies obtained due to docking procedure (with different theory level of optimization process) and the experimental binding affinities calculated for each compound are presented on the Fig. 11.

Analyses of Table 4 and Fig. 11 suggest that the PathDock approach is a more adequate method to study the free binding energy of STS-inhibitors with their

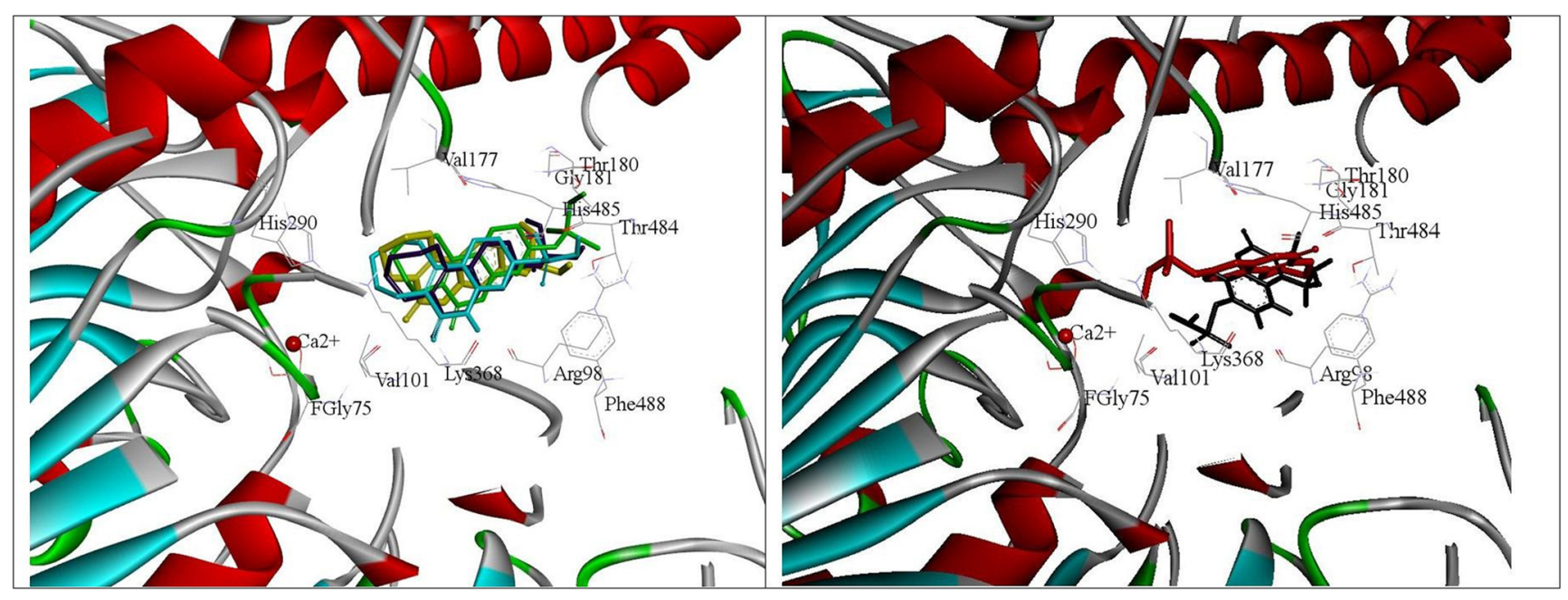

Fig. 9 Docked binding modes for the compounds $\mathbf{j}$ (Yellow), $\mathbf{k}$ (Purple), $\mathbf{m}$ (Green) and $\mathbf{s}$ (Cyan) in left and for the compounds $\mathbf{I}$ (Red) and COUMATE (Black) in right for PM7 method in PatchDock software 


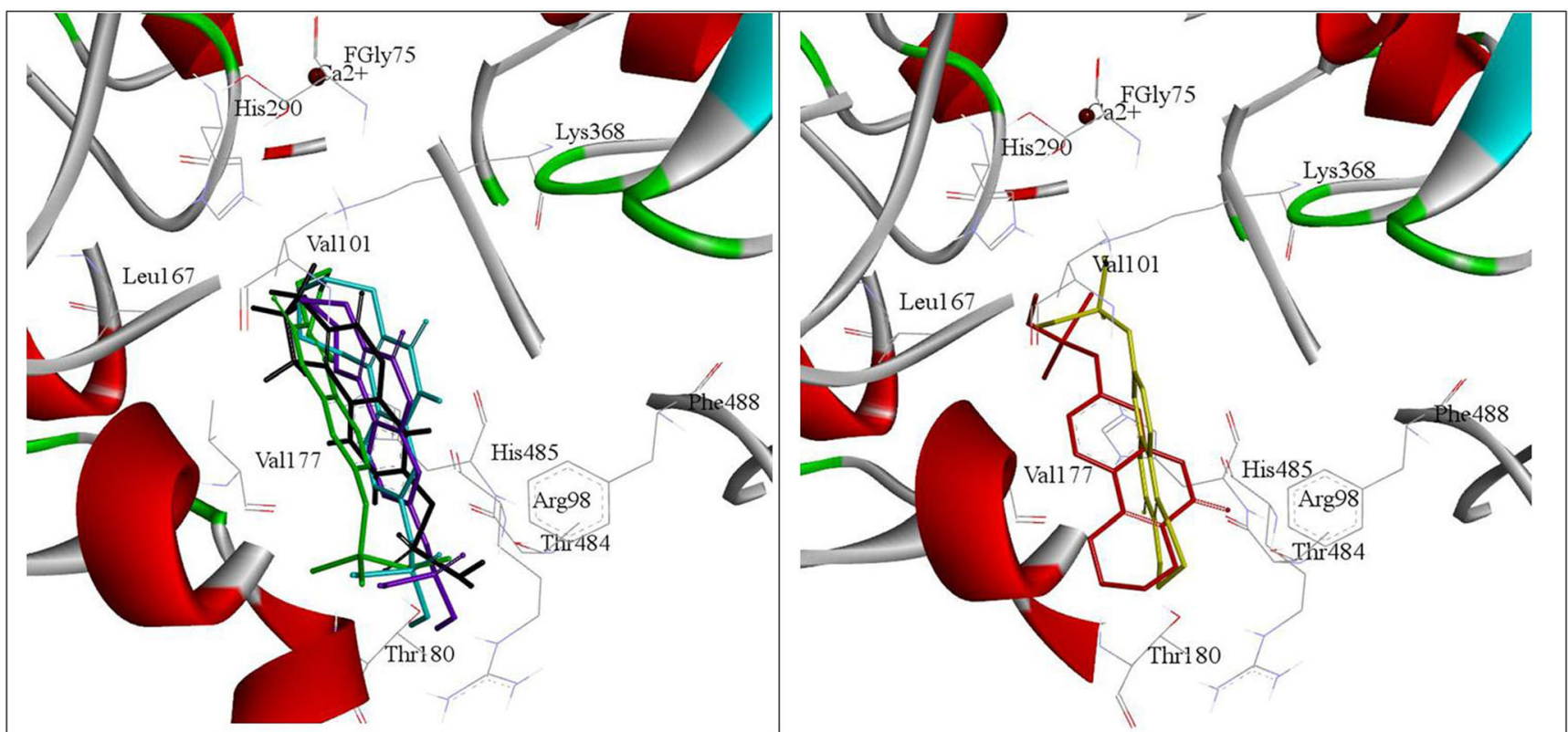

Fig. 10 Docked binding modes for the compounds $\mathbf{k}$ (Purple), $\mathbf{m}$ (Green), $\mathbf{s}$ (Cyan) and COUMATE (Black) in left and for the compounds $\mathbf{j}$ (Yellow) and $\mathbf{l}($ Red $)$ in right for DFT B3LYP/6-31++G* method in PatchDock software

molecular target. In the case of AutoDock the residuals between binding energies calculated by means of experimentally measuring the $\mathrm{IC}_{50}$ value and the ones computed in docking procedure are much more significant (e.g. residual for $\mathbf{m}$ compound optimized with $\mathrm{MM}+$ method docked by means of AutoDock is equal to almost $10 \mathrm{kcal} \mathrm{mol}^{-1}$, while in the case of PathDock it is only $2,67 \mathrm{kcal}^{*} \mathrm{~mol}^{-1}$ ). Thus, based on our results the
PathDock docking software is more recommended to study binding of STS-inhibitors with the STS protein.

According to the PathDock results, it was interesting to point out that the best agreement between the computed free binding energy and the experimentally measured ones (the lowest residuals observed in Fig. 11) is in the case of derivatives optimized with the MM+ method. However, differences in residuals obtained for compounds

Table 4 Molecular docking results

\begin{tabular}{|c|c|c|c|c|c|}
\hline ID & Optimization Method & $\Delta \mathrm{G}($ AuthoDock $)\left(\mathrm{kcal} \mathrm{mol}^{-1}\right)$ & $\Delta \mathrm{G}$ (PathDock) (kcal mol $\left.{ }^{-1}\right)$ & $\mathrm{IC} 50(\mu \mathrm{M})$ & $\Delta \mathrm{G}^{\mathrm{a}}\left(\mathrm{kcal} \mathrm{mol}^{-1}\right)$ \\
\hline \multirow[t]{3}{*}{$\mathrm{j}$} & $\mathrm{MM}+$ & -3.50 & -6.0 & \multirow[t]{3}{*}{37.8} & \multirow[t]{3}{*}{-6.03} \\
\hline & PM7 & -4.56 & -5.6 & & \\
\hline & B3LYP/631++G* & -3.96 & -7.0 & & \\
\hline \multirow[t]{3}{*}{$\mathrm{k}$} & $\mathrm{MM+}$ & -1.24 & -6.4 & \multirow[t]{3}{*}{21.5} & \multirow[t]{3}{*}{-6.36} \\
\hline & PM7 & -2.69 & -6.6 & & \\
\hline & B3LYP/631++G* & -1.74 & -6.7 & & \\
\hline \multirow[t]{3}{*}{1} & $\mathrm{MM}+$ & -0.03 & -7.7 & \multirow[t]{3}{*}{34.9} & \multirow[t]{3}{*}{-6.07} \\
\hline & PM7 & -2.98 & -8.7 & & \\
\hline & B3LYP/631++G* & -1.48 & -8.5 & & \\
\hline \multirow[t]{3}{*}{$\mathrm{m}$} & $\mathrm{MM}+$ & 2.90 & -8.7 & \multirow[t]{3}{*}{13.3} & \multirow[t]{3}{*}{-6.64} \\
\hline & PM7 & -1.03 & -9.6 & & \\
\hline & B3LYP/631++G* & 0.91 & -9.3 & & \\
\hline \multirow[t]{3}{*}{$\mathrm{s}$} & $\mathrm{MM}+$ & -0.91 & -6.0 & \multirow[t]{3}{*}{79.2} & \multirow[t]{6}{*}{-5.59} \\
\hline & PM7 & -3.55 & -6.1 & & \\
\hline & B3LYP/631++G* & -2.43 & -6.3 & & \\
\hline \multirow[t]{3}{*}{ 667-coumate } & $\mathrm{MM}+$ & -5.54 & -9.3 & \multirow[t]{3}{*}{-} & \\
\hline & PM7 & -5.51 & -9.5 & & \\
\hline & B3LYP/631++G* & -5.56 & -8.6 & & \\
\hline
\end{tabular}

${ }^{\mathrm{a}} \Delta \mathrm{G}$ - Free binding energy calculated according to the formula $\Delta \mathrm{G}=-\mathrm{RT} \ln \mathrm{K}_{\mathrm{i}}[44]$ 


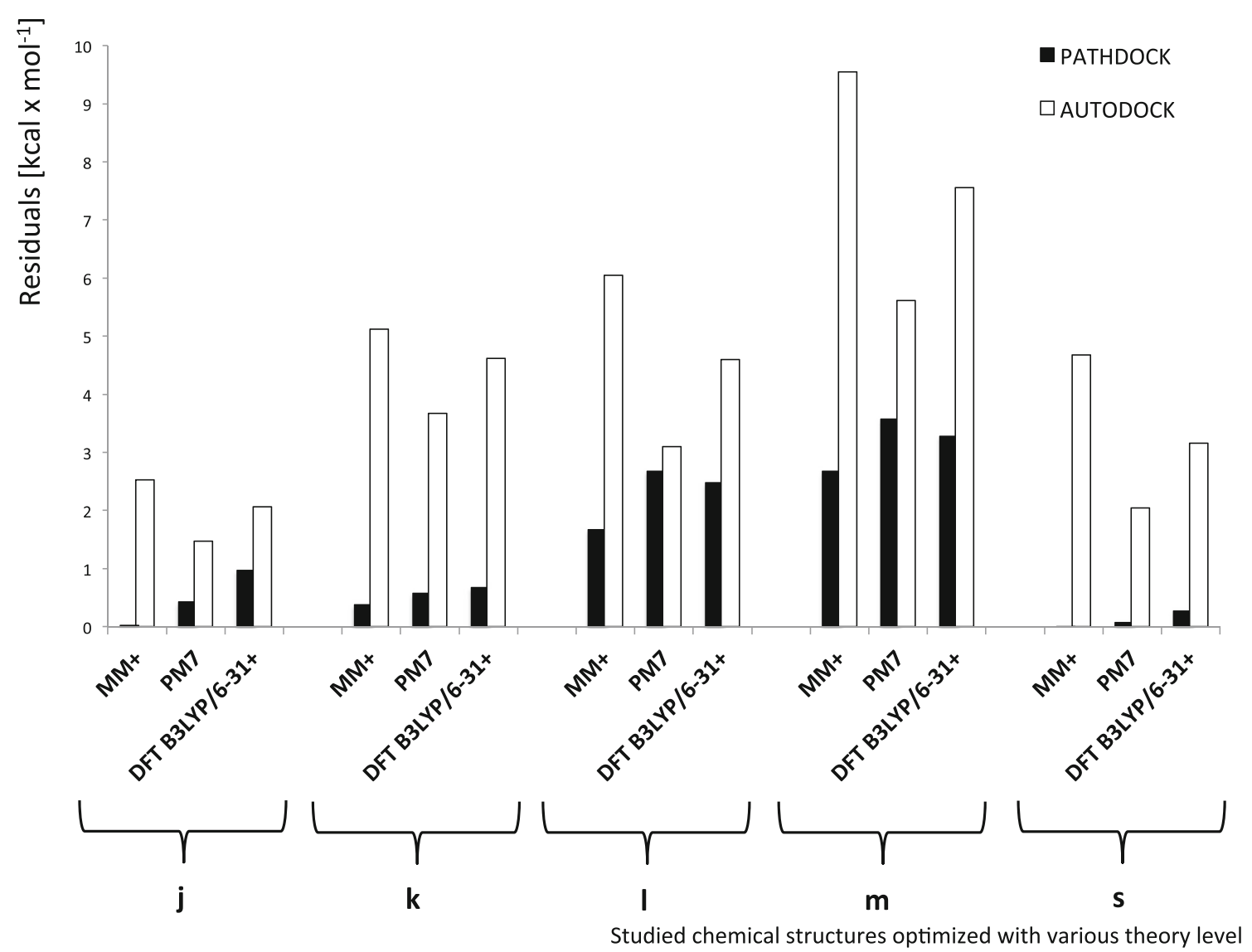

Fig. 11 Residuals between free binding energy calculated by means of experimentally measured $\mathrm{IC}_{50}$ and obtained in molecular docking

optimized with different approaches $(\mathrm{MM}+, \mathrm{PM} 7$ and DFT B3LYP/6-31++ $\mathrm{G}^{*}$ ) are, in the case of compounds $\mathbf{j}$, $\mathbf{k}$, and $\mathbf{s}$, negligible, which indicate that the method of geometry optimization is not the crucial factor in free binding energy computation by means of docking procedure. This is reasonable taking into account that docking simulation includes exploration of the best conformation of the flexible ligand (the energetically most favourable) to increase the energy of the ligand-receptor interaction $[33,48]$. Therefore, changes in ligand structure during docking simulation are possible.

The more important factor determining the free binding energy of STS-inhibitors with the STS protein is ligand orientation that influences their free binding energy. In almost all performed docking simulations, compounds $\mathbf{I}$ and $\mathbf{m}$ bind with the STS protein in different ways, having opposite orientation in comparison to other derivatives, regardless of the applied method of geometry optimization. Moreover, the residuals between the experimentally measured and the computed free for these two derivatives (l and $\mathbf{m}$ ), regardless of the applied method of geometry optimization and docking procedure are the highest, which in fact confirms that the orientation opposite to the reference compounds is not adequate.

\section{Conclusions}

In the presented work, we have evaluated the influence of the method of geometry optimization on the geometry expression in bond lengths, angles and dihedral angles of steroid sulfatase inhibitors. We have employed two methods that differ in theory levels, such as: molecular mechanics (MM+) and quantum mechanics (DFT B3LYP/6-31++G* and PM7). The obtained results indicate that application of these techniques allows obtaining different conformers. Surprisingly, we have revealed that the selection of the geometry optimization method did not affect the optimal STS inhibitor coordinates, as well as the values of the molecular descriptors that describe the 3D structure of the obtained conformers.

Additionally, we have verified the impact of the geometry optimization method on the free binding energy of steroid sulfatase inhibitors with the STS protein. The results indicate that the geometry optimization did not influence significantly the binding of these compounds with their molecular target. More crucial factors include: (i) selection of the software to molecular docking; and (ii) proper orientation of ligand into its binding pocket.

Thus, taking into account that the time and computational cost required to perform calculation with $\mathrm{MM}+$ or PM7 method are much less demanding than for the DFT one, these 
methods would be recommended to optimize geometries of STS inhibitors before their docking procedure, as well as for molecular descriptor calculations.

Acknowledgements The authors (K. J., A. S. and T. P.) thank the Polish Ministry of Science and Higher Education (grant no. DS 530-8637-D510-15/ 16). We also would like to thank our colleague Maciej Barycki from University of Gdańsk for writing the Matlab code that allowed performing the Wilcoxon test for the large dataset. Calculations were carried out at the Academic Computer Center in Gdansk. S. K. and J. L. thank the National Science Foundation (NSF/CREST HRD-1547754, and EPSCoR (award no. 362492-190200-01/NSFEPS-090378) for the financial support.

Open Access This article is distributed under the terms of the Creative Commons Attribution 4.0 International License (http:// creativecommons.org/licenses/by/4.0/), which permits unrestricted use, distribution, and reproduction in any medium, provided you give appropriate credit to the original author(s) and the source, provide a link to the Creative Commons license, and indicate if changes were made.

\section{References}

1. Ireson CR, Chander SK, Purohit A, Parish DC, Woo LWL, Potter BVL, Reed MJ (2004) Pharmacokinetics of the nonsteroidal steroid sulphatase inhibitor 667 COUMATE and its sequestration into red blood cells in rats. Brit J Cancer 91(7):1399-1404. doi:10.1038/sj. bjc. 6602130

2. Demkowicz S, Kozak W, Dasko M, Maslyk M, Kubinski K, Rachon J (2015a) Phosphate and thiophosphate biphenyl analogs as steroid sulfatase inhibitors. Drug Develop Res 76(2):94-104. doi: $10.1002 / \mathrm{ddr} .21245$

3. Reed MJ, Purohit A, Woo LWL, Newman SP, Potter BVL (2005) Steroid sulfatase: molecular biology, regulation, and inhibition. Endocr Rev 26(2):171-202. doi:10.1210/er.2004-0003

4. Woo LWL, Howarth NM, Purohit A, Hejaz HAM, Reed MJ, Potter BVL (1998) Steroidal and nonsteroidal sulfamates as potent inhibitors of steroid sulfatase. J Med Chem 41(7):1068-1083. doi:10.1021/Jm970527v

5. Malini B, Purohit A, Ganeshapillai D, Woo LWL, Potter BVL, Reed MJ (2000) Inhibition of steroid sulphatase activity by tricyclic coumarin sulphamates. J Steroid Biochem 75(4-5):253-258. doi:10.1016/S0960-0760(00)00178-3

6. Demkowicz S, Rachon J, Dasko M, Kozak W (2016a) Selected organophosphorus compounds with biological activity. Applications in medicine. RSC Adv 6(9):7101-7112. doi:10.1039 /c5ra25446a

7. Demkowicz S, Kozak W, Dasko M, Maslyk M, Gielniewski B, Rachon J (2015b) Synthesis of bicoumarin thiophosphate derivatives as steroid sulfatase inhibitors. Eur J Med Chem 101:358-366. doi:10.1016/j.ejmech.2015.06.051

8. Kozak W, Dasko M, Maslyk M, Pieczykolan JS, Gielniewski B, Rachon J, Demkowicz S (2014) Phosphate tricyclic coumarin analogs as steroid sulfatase inhibitors: synthesis and biological activity. RSC Adv 4(84):44350-44358. doi:10.1039/c4ra07135b

9. Kozak W, Dasko M, Maslyk M, Gielniewski B, Rachon J, Demkowicz S (2015) Synthesis and biological evaluation of thiophosphate tricyclic coumarin derivatives as steroid sulfatase inhibitors. J Asian Nat Prod Res 17(11):1091-1096. doi:10.1080 $/ 10286020.2015 .1054815$

10. Demkowicz S, Dasko M, Kozak W, Krawczyk K, Witt D, Maslyk M, Kubinski K, Rachon J (2016b) Synthesis and biological evaluation of fluorinated 3-phenylcoumarin-7-O-sulfamate derivatives as steroid sulfatase inhibitors. Chem Biol Drug Des 87(2): 233-238. doi:10.1111/cbdd.12652

11. Mandal S, Moudgil M, Mandal SK (2009) Rational drug design. Eur J Pharmacol 625(1-3):90-100. doi:10.1016/j. ejphar.2009.06.065

12. Hamzeh-Mivehroud M, Sokouti, B., Dastmalchi, S. (2015) An introduction to the basic concepts in QSAR - aided drug design. In: K. R (ed) Quantitative structure-activity relationships in drug design, predictive toxicology and risk assessment. IGI Global, Hershey PA, p 1-47

13. Sliwoski G, Kothiwale S, Meiler J, Lowe Jr EW (2014) Computational methods in drug discovery. Pharmacol Rev 66(1): 334-395. doi:10.1124/pr.112.007336

14. Kubinyi H, Bohm HJ (1997) Computer-aided drug design: current state and future perspectives. Abstr Pap Am Chem S 214:44-CINF

15. Anderson AC (2003) The process of structure-based drug design. Chem Biol 10(9):787-797. doi:10.1016/j.chembiol.2003.09.002

16. Gane PJ, Dean PM (2000) Recent advances in structure-based rational drug design. Curr Opin Struc Biol 10(4):401-404. doi:10.1016/S0959-440x(00)00105-6

17. Ou-Yang SS, Lu JY, Kong XQ, Liang ZJ, Luo C, Jiang HL (2012) Computational drug discovery. Acta Pharmacol Sin 33(9):11311140. doi:10.1038/aps.2012.109

18. Roy K (2015) Quantitative structure-activity relationships in drug design, predictive toxicology and risk assessment. IGI Global, Hershey PA

19. Yang SY (2010) Pharmacophore modeling and applications in drug discovery: challenges and recent advances. Drug Discov Today 15(11-12):444-450. doi:10.1016/j.drudis.2010.03.013

20. Meng XY, Zhang HX, Mezei M, Cui M (2011) Molecular docking: a powerful approach for structure-based drug discovery. Curr Comput-Aid Drug 7(2):146-157

21. Schrodinger Suite (2016) QM-Polarized Ligand Docking protocol, https://www.schrodinger.com/qmpolarized-ligand-docking

22. Kubinyi H (1997a) QSAR and 3D QSAR in drug design .1. Methodology. Drug Discov Today 2(11):457-467. doi:10.1016 /S1359-6446(97)01079-9

23. Kubinyi H (1997b) QSAR and 3D QSAR in drug design .2. Applications and problems. Drug Discov Today 2(12):538-546. doi:10.1016/S1359-6446(97)01084-2

24. Dennington RKT, Millam J (2009) GaussView, 5th edn. Semichem Inc., Shawnee Mission, Kansas

25. Toropov AA, Toropova AP (2015) Quasi-SMILES and nanoQFAR: united model for mutagenicity of fullerene and MWCNT under different conditions. Chemosphere 139:18-22. doi:10.1016 /j.chemosphere.2015.05.042

26. Stewart J (2012) MOPAC20012. Stewart Computational Chemistry, Colorado Springs

27. Lee C, Yang W, Parr RG (1988) Development of the Colle-Salvetti correlation-energy formula into a functional of the electron density. Phys Rev B Condens Matter 37(2):785-789

28. Becke AD (1988) Density-functional exchange-energy approximation with correct asymptotic behavior. Phys Rev A 38(6):30983100

29. Mclean AD, Chandler GS (1980) Contracted Gaussian-basis sets for molecular calculations .1. 2nd row atoms, $Z=11-18$. J Chem Phys 72(10):5639-5648. doi:10.1063/1.438980

30. Krishnan R, Binkley JS, Seeger R, Pople JA (1980) Self-consistent molecular-orbital methods. 20. Basis set for correlated wave-functions. J Chem Phys 72(1):650-654. doi:10.1063/1.438955

31. Frisch MJT, Trucks GW, Schlegel HB, Scuseria GE, Robb MA, Cheeseman JR, Scalmani G, Barone V, Mennucci B, Petersson GA, Nakatsuji H, Caricato M, Li X, Hratchian HP, Izmaylov AF, Bloino J, Zheng G, Sonnenberg JL, Hada M, Ehara M, Toyota K, Fukuda R, Hasegawa J, 
Ishida M, Nakajima T, Honda Y, Kitao O, Nakai H, Vreven T, Montgomery JA Jr, Peralta JE, Ogliaro F, Bearpark M, Heyd JJ, Brothers E, Kudin KN, Staroverov VN, Kobayashi $\mathrm{R}$, Normand J, Raghavachari K, Rendell A, Burant JC, Iyengar SS, Tomasi J, Cossi M, Rega N, Millam JM, Klene M, Knox JE, Cross JB, Bakken V, Adamo C, Jaramillo J, Gomperts R, Stratmann RE, Yazyev O, Austin AJ, Cammi R, Pomelli C, Ochterski JW, Martin RL, Morokuma K, Zakrzewski VG, Voth GA, Salvador P, Dannenberg JJ, Dapprich S, Daniels AD, Farkas Ö, Foresman JB, Ortiz JV, Cioslowski J, Fox DJ (2009) Gaussian 09, revision A.02. Gaussian, Inc, Wallingford CT

32. Talete (2014) Dragon (software for molecular descriptor calculation http://www.talete.mi.it/

33. Morris GM, Huey R, Lindstrom W, Sanner MF, Belew RK, Goodsell DS, Olson AJ (2009) AutoDock4 and AutoDockTools4: automated docking with selective receptor flexibility. J Comput Chem 30(16):2785-2791. doi:10.1002/jcc.21256

34. Woo LW, Fischer DS, Sharland CM, Trusselle M, Foster PA, Chander SK, Di Fiore A, Supuran CT, De Simone G, Purohit A, Reed MJ, Potter BV (2008) Anticancer steroid sulfatase inhibitors: synthesis of a potent fluorinated second-generation agent, in vitro and in vivo activities, molecular modeling, and protein crystallography. Mol Cancer Ther 7(8):2435-2444. doi:10.1158/1535-7163. MCT-08-0195

35. Schneidman-Duhovny D, Inbar Y, Nussinov R, Wolfson HJ (2005) PatchDock and SymmDock: servers for rigid and symmetric docking. Nucleic Acids Res 33:W363-W367. doi:10.1093 /nar/gki481

36. Zhao WW, Bian WS (2007) Investigation of the structures and electronic spectra for coumarin-6 through TD-DFT calculations including PCM solvation. J Mol Struc-Theochem 818(1-3):43-49. doi:10.1016/j.theochem.2007.05.002

37. Zhao WW, Bian WS (2008) Investigation of the structures and electronic spectra of two coumarins with heterocyclic substituents through TD-DFT calculations. J Mol Struc-Theochem 859(1-3): 73-78. doi:10.1016/j.theochem.2008.03.004

38. Momany FA, Willett JL (2000) Computational studies on carbohydrates: I. Density functional ab initio geometry optimization on maltose conformations. J Comput Chem 21(13):1204-1219. doi:10.1002 /1096-987x(200010)21:13<1204::Aid-Jcc9>3.3.Co;2-6

39. Vreven T, Morokuma K, Farkas O, Schlegel HB, Frisch MJ (2003) Geometry optimization with QM/MM, ONIOM, and other combined methods. I. Microiterations and constraints. J Comput Chem 24(6):760-769. doi:10.1002/jcc.10156

40. Duan Y, Wu C, Chowdhury S, Lee MC, Xiong GM, Zhang W, Yang R, Cieplak P, Luo R, Lee T, Caldwell J, Wang JM, Kollman P (2003) A point-charge force field for molecular mechanics simulations of proteins based on condensed-phase quantum mechanical calculations. J Comput Chem 24(16): 1999-2012. doi:10.1002/jcc.10349

41. Morozov AV, Misura KMS, Tsemekhman K, Baker D (2004) Comparison of quantum mechanics and molecular mechanics dimerization energy landscapes for pairs of ring-containing amino acids in proteins. J Phys Chem B 108(24):8489-8496. doi:10.1021/jp037711e

42. Shigeta Y (2004) Hybrid QM/MM studies on energetics of malonaldehyde in condensed phase. Int J Quantum Chem 96(1):32-41. doi:10.1002/qua.10790

43. Ward JH (1963) Hierarchical grouping to optimize an objective function. J Am Stat Assoc 58(301):236-\&. doi:10.2307/2282967

44. Durdagi S, Mavromoustakos T, Chronakis N, Papadopoulos MG (2008) Computational design of novel fullerene analogues as potential HIV-1 PR inhibitors: analysis of the binding interactions between fullerene inhibitors and HIV-1 PR residues using 3D QSAR, molecular docking and molecular dynamics simulations. Bioorgan Med Chem 16(23):9957-9974. doi:10.1016/j. bmc.2008.10.039

45. Tzoupis H, Leonis G, Durdagi S, Mouchlis V, Mavromoustakos T, Papadopoulos MG (2011) Binding of novel fullerene inhibitors to HIV-1 protease: insight through molecular dynamics and molecular mechanics Poisson-Boltzmann surface area calculations. J Comput Aided Mol Des 25(10):959-976. doi:10.1007/s10822-011-9475-4

46. Sengupta D, Verma D, Naik PK (2007) Docking mode of delvardine and its analogues into the p66 domain of HIV-1 reverse transcriptase: screening using molecular mechanics-generalized born/surface area an absorption, distribution, metabolism and excretion properties. J Biosci 32(7):1307-1316

47. Conn PJ, Sanders-Bush E, Hoffman BJ, Hartig PR (1986) A unique serotonin receptor in choroid plexus is linked to phosphatidylinositol turnover. Proc Natl Acad Sci USA 83:4086-4088

48. Morris GM, Huey R, Lindstrom W, Li CL, Zhao Y, Hart WE, Belew R, Sanner MF, Goodsell DS, Olson WJ (2004) Recent advances in autodock: search, representation and scoring. Abstr Pap Am Chem S 228:U508-U508 